\section{Characteristics of human subepicardial adipose tissue-derived stem cells}

\author{
Tatjana A. Krylova, ${ }^{1}$ Olga A. Bystrova, ${ }^{1}$ \\ Tatjana K. Jakovleva, ${ }^{1}$ Valerij V. Zenin, ${ }^{1}$ \\ Olga M. Moiseeva, ${ }^{2}$ Vladimir N. Parfenov, \\ Marina G. Martynova' \\ ${ }^{1}$ Institute of Cytology, Russian Academy \\ of Sciences; ${ }^{2}$ Research Institute of \\ Cardiology, Russian Federation Ministry \\ of Health, St. Petersburg, Russia
}

\section{Abstract}

Here, we report on the isolation and characterization of human subepicardial adipose tissue-derived cells with stem cell properties (hcADSCs). The morphology, proliferative activity, karyotype, phenotype, and differentiation potential of these cells at early and late passages were analyzed. Cytogenetic analysis showed that after five passages hcADSCs retained a genetic stability while passage 12 cells exhibited a number of genetic abnormalities such as chromosomal aneuploidy and multi-ploidy karyotypes. Immunophenotyping revealed that the passage 5 hcADSCs lacked expression of the hematopoietic line markers CD34 and HLA class II, and cancer stem cell marker CD133, but were strongly positive for mesenchymal stem cells markers CD13, CD44, and CD73, and CD90. These cells were also moderately positive for CD11b, CD41, CD45, CD71, CD117, CD130, and HLA class I antigens. The hcADSC surface marker profile changed by passage 16 showing decrease in CD41 and increases in CD 117 expression. Cultured hcADSCs were capable of both spontaneous and directed differentiation. By culturing without any induction factors, hcADSC population contained the cells exhibiting the markers characteristic of endothelial cells and myocytes, and at late passages also of adipocytes. In appropriate induction media, hcADSCs could also be differentiated into osteogenic and chondrogenic lineages. Our findings suggest that human subepicardial adipose tissue contains the resident capable of self-renewal cell population which represents a heterogeneous mixture of progenitor cells with varied differentiation potential. These cells possess a number of specific phenotypic and differentiation characteristics. Revealed peculiar properties of hcADSCs might make them a promising candidate for cellular heart therapy.

\section{Introduction}

Following first studies on isolation and characterization of mesenchymal stem cells (MSCs) from bone marrow, ${ }^{1,2}$ MSCs have been also identified in many other adult organs and tissues, including skin, ${ }^{3}$ liver, ${ }^{4}$ skeletal muscle ${ }^{5}$ brain, pancreas, ${ }^{6}$ heart, ${ }^{7}$ and spleen. ${ }^{8}$ MSCs are mainly characterized by their ability to proliferate and differentiate into distinct cell types of the mesodermal lineage including adipocytes, osteocytes, chondrocytes, and myocytes. Exciting information has recently emerged to show that subcutaneous fat may be perspective source of MSCs. Adipose tissue is widely scattered around the body. Apart from its importance for the mechanical and energy providing functions, adipose tissues are known to participate in many significant physiological processes. There is accumulating evidence that adipocytes secrete a number of cytokines acting in a paracrine or autocrine fashion. The cells with stem cell characteristics were isolated from subcutaneous, ${ }^{9-11}$ perirenal, ${ }^{8}$ periumbilical, ${ }^{12,13}$ and omental ${ }^{14}$ adipose tissues. Adipose tissue-derived stem cells from different depots differ in surface marker profile. Such differences have been revealed between periumbilical and eyelid fat, ${ }^{12}$ or between omental and subcutaneous fat. ${ }^{14}$

Adipose tissue is also associated with the epicardial surface of the heart and coronary great vessels, and constitutes about $20 \%$ of total heart weight. ${ }^{15}$ Subepicardial adipose tissue shares many features with adipose tissues of other localization. However, there are significant differences in morpho-functional properties between the epicardial adipose tissue and other fat depots in the body, which include size of adipocytes, fatty acid and protein content, the rate of metabolism and others. ${ }^{15}$ Taken together these data hint at a possibility that subepicardial adipose tissue as a stem cell niche provides a special unique microenvironment for the resident stem cells, and determine their peculiar characteristics.

Recently the lineage of MSC-like cells was obtained from human adult cardiac adipose tissue. ${ }^{16}$ The authors showed the capacity of these cells to improve cardiac function when transplanted into injured myocardium in rodent models of myocardial infarction. These cells were found to migrate to ischemic areas and promote local vascularization. Moreover, their potential to differentiate into cardiac and endothelial cell lineages in vitro was demonstrated.

However, the human cardiac adipose tissuederived stem cells (hcADSCs) have not been described in sufficient details. Here, to establish basic characteristics of hcADSCs, we examined their morphology, proliferative activity, karyotype, and expression of surface markers over long-term passages. Furthermore, we evaluated their capacity to differentiate into multiple cell lineages.
Correspondence: Marina G. Martynova, Institute of Cytology RAS, Tikhoretsky Ave, 4, St,Petersburg 194064, Russia. Fax - (812)2790341. E-mail: heartdev@mail.cytspb.rssi.ru

Key words: stem cells, karyotype, phenotype, spontaneous and induced differentiation.

Acknowledgments: the authors would like to thank the granting program Molecular and Cell Biology of Russian Academy of Sciences, in particular Elena Nakatseva for providing the cardiac fat tissue samples, and to Olga Emeljanova for technical assistance.

Contributions: TK, culturing and differentiation experiments; OB, immunohistochemistry; TJ, karyotyping; VZ, phenotyping; OM, provision of study material, scientific consultations; VP, scientific consultations, administrative support; MM, data analysis and interpretation, manuscript writing.

Conflict of interests: the authors report no conflicts of interest.

Received for publication: 31 August 2011.

Revision received: 17 August 2011.

Accepted for publication: 19 October 2011.

This work is licensed under a Creative Commons Attribution NonCommercial 3.0 License (CC BYNC 3.0).

(C) Copyright T.A. Krylova et al., 2011

Licensee PAGEPress, Italy

Stem Cell Studies 2011; 1:e18

doi:10.4081/scs.2011.e18

\section{Materials and Methods}

\section{Cell culture}

Fat samples were taken at open heart surgery from three male patients (aged 45 to 50 years) who have undergone coronary artery bypass grafting. All studies were carried out in accordance with the declaration of Helsinki, with local hospital ethics committee approval. Biopsy (10-15 g) from subepicardial adipose tissue localized in the atrioventricular groove of the left heart was taken with scissors before connecting the equipment for artificial blood circulation. Tissue samples have been transported within $1 \mathrm{~h}$ into lab in serum-free DMEM (GIBCO, Invitrogen Corp., Carlsbad, CA, USA) supplemented with $50 \mu \mathrm{g} / \mathrm{mL}$ gentomycin (Sigma Aldrich Corp., St. Louis, Mo, USA). After a careful washing with calcium- and magnesium-free phosphate buffered saline (PBS), the samples were mechanically trimmed and enzymatically dissociated with $0.1 \%$ collagenase type I (GIBCO) for $40 \mathrm{~min}$ at 
room temperature. Digestion enzyme activity was neutralized with fetal bovine serum (FBS) (HyClone). The cell suspension was centrifuged at $900 \times \mathrm{g}$ for $7 \mathrm{~min}$, and obtained pellet was then resuspended in growth medium aMEM (Sigma) supplemented with 10\% FBS, $100 \mathrm{U} / \mathrm{mL}$ penicillin, $100 \mathrm{U} / \mathrm{mL}$ streptomycin, and $2 \mathrm{mM}$ glutamin (all from GIBCO), filtered through a $100-\mu \mathrm{m}$ nylon mesh to remove cell debris, and centrifuged once more. Then the cells were routinely seeded at a density of $3 \times 10^{4} \mathrm{cells} / \mathrm{cm}^{2}$ and cultured in Petri dishes or culture flasks at $37^{\circ} \mathrm{C}$ in a humidified atmosphere containing $5 \% \mathrm{CO}_{2}$. After $48 \mathrm{~h}$, the cells started to adhere to the bottom of the culture well. The medium was changed twice a week.

\section{Cell proliferation}

The proliferation characteristics of hcADSCs at different culture passages were examined using immunostaining with antiPCNA (Proliferating Cell Nuclear Antigen) antibody. The cells were fixed with $4 \%$ paraformaldehyde (PFA) at room temperature for $15 \mathrm{~min}$ at passages 6,10 and 19 one day after seeding. The proportion of PCNA-positive cells was estimated from 1000 cells for each population.

\section{Electron microscopy and immuno- gold labeling}

For transmission electron microscopy, cells adhered to the coverslips or suspension of cells detached with trypsin/EDTA were fixed in 2.5\% glutaraldehyde in $0.1 \mathrm{M}$ cacodylate buffer, $\mathrm{pH}$ 7.2-7.4, for $1 \mathrm{~h}$ at $4^{\circ} \mathrm{C}$, postfixed in $1 \%$ aqueous $\mathrm{OsO}_{4}$ for $1 \mathrm{~h}$, dehydrated, and embedded in Epon and Araldit, and then sectioned with a diamond knife on a LKB ultratome. Ultrathin sections were collected on fine mesh copper or nickel grids, and stained with uranyl acetate and lead citrate for examination with Jem 7A electron microscope operating at $80 \mathrm{kV}$.

For EM immunocytochemical analysis, ultrathin sections mounted on nickel grids were first treated with hydrogen peroxide for $20 \mathrm{~min}$ to loosen the resin. Three washes in PBS for 2 min each were followed by incubation with mouse anti-collagen type II monoclonal antibody (Chemicon, Temecula, CA, USA) diluted 1:2000 in 0.05 M Tris-HCl buffer, $\mathrm{pH} 7.4$, containing $1 \%$ BSA and $0.1 \%$ coldwater fish gelatin overnight, at $4^{\circ} \mathrm{C}$ in a moist chamber. Goldconjugated (10nm) goat anti-mouse IgG (Sigma) diluted 1:10 was used as the secondary antibody and sections were incubated for 1 $\mathrm{h}$ at room temperature. Finally, the sections were counterstained as described. For the control, the primary antibody was omitted or replaced by irrelevant antibodies.

\section{Flow cytometric analysis}

The hcADSCs at culture passages 5 and 16 were resuspended and incubated in PBS con- taining fluorescein isothiocyanate (FITC)- or phycoerythrin (PE)-coupled antibodies against CD10, CD11b, CD13, CD34, CD41, CD44, CD45, CD71, CD73, CD90, CD105, CD117, CD130, and CD133 as well as HLA class I and HLA class II. All antibodies were obtained from BD Biosciences. After incubation for $30 \mathrm{~min}$ at $4^{\circ} \mathrm{C}$ in the dark, cells were resuspended in $1 \mathrm{~mL}$ PBS containing 2 mM EDTA for immunophenotypical investigation. Labeled cells were analyzed by flow cytomety in a Coulter Epics XL (Beckman Coulter Inc., Brea, CA, USA) and data were analysed with the CellQuest software (Becton Dickinson, Bedford, MA USA).

\section{Cytogenetic analysis}

The cytogenetic analysis of hcADSCs at passages 5 and 12 was performed according to standard technique. After exposure to mitotic arrestant colchicine (final concentration of $0.05 \mu \mathrm{g} / \mathrm{mL}$ ) added to the culture medium for 40-60 min, cells were dislodged from the flask using the mix of trypsin and versene in PBS, resuspended by gentle mixing, and collected by centrifugation. The hypotonic effect was attained by the addition of $0.075 \mathrm{M} \mathrm{KCl}$ and $1 \%$ sodium citrate (1:1) for 15-20 min. Then cells run 3 times, each time $10 \mathrm{~min}$, through a fixative (methanol-acetic acid, 3:1). Metaphase spreads were prepared by a standard air-dry procedure. G-banding was obtained according to the method of Ozkiney, Mitelman (1979). ${ }^{17}$ Metaphase chromosomes were observed with a microscope Axio Imager.M1 (Carl Zeiss, Oberkochen, Germany) equipped with Ikaros4 Karyotyping System (MetaSystems, Altlussheim, Germany). At least 100 metaphases for each passage were analyzed for determination of the modal number of chromosomes. The proportion of polyploid cells was estimated from 850 metaphases at passage 5 , and from 1000 metaphases at passage 12 . Thirty-two and 25 metaphases were karyotyped for passages 5 and 12 , respectively, and described according to the International System for Human Cytogenetic Nomenclature (ISCN, 2009). ${ }^{18}$

\section{Antibodies and immunocytochem- istry}

A standard immunocytochemistry protocol was used. Cells on the coverslips were fixed with 4\% PFA at room temperature for $15 \mathrm{~min}$ followed by three washes with PBS for $5 \mathrm{~min}$, and then treated with $0.1 \%$ Triton $\mathrm{X}-100$ for 10 min to increase permeability of the cell membranes. After three washes in PBS containing 0.2\% Tween 20 for $5 \mathrm{~min}$, cells were incubated at room temperature for 10 min in $3 \% \mathrm{H}_{2} \mathrm{O}_{2}$ to block endogenous peroxidase, and for 20 min in $10 \%$ FBS (Sigma) to block non-specific binding of antibodies. Then, cells were incubated with first antibodies overnight at $4^{\circ} \mathrm{C}$. The following primary antibodies were used: mouse anti-PCNA monoclonal antibody (Proliferating Cell Nuclear Antigen, 1:1000 dilution; Sigma), mouse antiCD31 monoclonal antibody (1:50 dilution; BD Biosciences, Franklin Lakes, NJ, USA), rabbit anti-vWF polyclonal antibody (von Willebrand's factor, 1:20 dilution; Abcam, Cambridge, MA, USA), mouse anti-VCAM-1 monoclonal antibody (vascular cellular adhesion molecule-1, 1:50 dilution; Novacastra, Leica Microsystems, Wetzlar, Germany), mouse anti-hSMA monoclonal antibody (human Smooth Muscle Actin, 1:100 dilution; DAKOCytomation, Glostrup, Denmark), chondroitin sulfate monoclonal antibody (1:50 dilution; Sigma), and mouse anti-collagen type II monoclonal antibody (dilution 1:50: Chemicon; with pretreatment of the cells with $0.1 \%$ pepsin in TRIS buffer, $\mathrm{pH} 2.0$, for $15 \mathrm{~min}$ at room temperature). After washing with PBS, the mix of biotinylated anti-mouse and anti-rabbit IgG antibody (DAKO) was added and incubated for $20 \mathrm{~min}$ at room temperature. Then cells were treated with extrAvidin-peroxidase (dilution 1:400; Sigma) for 20 min. Cells were stained with $\mathrm{DAB}+$ chromogen (DAKO) for $3 \mathrm{~min}$ and hematoxylin before visualization. Proper controls for secondary antibodies revealed no nonspecific staining. Images were obtained using a light microscope Axiovert $200 \mathrm{M}$ (Zeiss) with the digital camera Leica DFC 420C.

\section{Differentiation assays}

The hcADSCs were analysed for their capacity to differentiate toward endothelial, adipogenic, chondrogenic, and osteogenic lineages using specific protocols.

\section{Endothelial differentiation}

Passage 6 hcADSCs were expanded by culture in growth medium. Once the cells reached confluency, they were detached with Trypsin/EDTA (Sigma), centrifuged, resuspended, and replated at the density of $1 \times 10^{5}$ per mL onto clear glass coverslips or onto the glass coverslips precoated with Matrigel (Becton Dickinson) $7.9 \mathrm{mg} / \mathrm{mL}$. Then the cells were induced to endothelial differentiation according to the procedure described by Cao et al..$^{19}$ In brief, the cells were cultured for 48-72 h in Medium 199 supplemented with 50 $\mathrm{ng} / \mathrm{mL}$ vascular endothelial growth factor (VEGF), $10 \mathrm{ng} / \mathrm{mL}$ basic fibroblast growth factor (bFGF), and $3 \% \mathrm{FBS}$ at $37^{\circ} \mathrm{C}$ in a $5 \% \mathrm{CO}_{2}$ atmosphere. Endothelial differentiation induction was assayed using immunohystochemical reactions with antibodies against CD31, vWF, and VCAM. Additionally, the ultrastructure of these cells was examined by electron microscopy.

\section{Adipogenesis}

For adipogenic induction the hcADSCs at passage 12 were plated at $2 \times 10^{4}$ cells $/ \mathrm{cm}^{2}$ and cultured for 2 to 3 weeks in differentiation medium. The adipogenic medium comprised of $\alpha M E M$, containing 10\% FBS, $50 \mu \mathrm{g} / \mathrm{mL}$ gentamycin, 10-9 
M dexamethasone (Sigma), $50 \mu \mathrm{g} / \mathrm{mL}$ ascorbate, and 1xITS (100x solution of $25 \mu \mathrm{g} / \mathrm{mL}$ insulin, 25 $\mu \mathrm{g} / \mathrm{mL}$ transferrin, and $25 \mu \mathrm{g} / \mathrm{mL}$ sodium selenite, Invitrogen). The medium was replaced three times a week. After 3 weeks, to conform adipogenesis, the cells were rinsed with PBS, fixed in absolute methanol for $2 \mathrm{~min}$ at $-20^{\circ} \mathrm{C}$, and stained with fresh $0.5 \%$ Oil red-0-solution in isopropanol for $10 \mathrm{~min}$ to visualize lipid vacuoles. Adipogenic differentiation was also visualized by the presence of numerous intracellular lipid droplets using electron microscopy.

\section{Chondrogenesis}

For chondrogenic induction, suspension of hcADSCs (passage 12) in serum-free medium, $5 \times 10^{5} \mathrm{cell} / \mathrm{mL}$, was collected in a conical bottom tube and centrifuged at $900 \times$ g for 5-7 min to form a pellet. The pellets were cultured in $0.5 \mathrm{~mL}$ chondrogenic differentiation medium consisting of DMEM HG (Invitrogen) supplemented with 10-7 dexamethasone (Sigma), $50 \mu \mathrm{g} / \mathrm{mL}$ ascorbate (ICN), and 1xITS [100x solution of $25 \mu \mathrm{g} / \mathrm{mL}$ insulin, 25 $\mu \mathrm{g} / \mathrm{mL}$ transferring, and $25 \mu \mathrm{g} / \mathrm{mL}$ sodium selenite (Invitrogen)] in the presence of 5 $\mathrm{ng} / \mathrm{mL}$ transforming growth factor-1 (TGF $\beta$-1) and $110 \mathrm{mg} / \mathrm{mL}$ sodium pyruvate. The medium was replaced every 3 days for up to 21 days. To detect chondrogenic differentiation, randomLy selected pellets were fixed in $4 \%$ phosphate-buffered formalin for $24 \mathrm{~h}$, embedded in paraffin, sectioned at $3 \mu \mathrm{m}$, and stained with Alcian blue ( $\mathrm{pH} 1.0)$. For immunohistochemistry, the sections were deparaffinized in xylene, rehydrated, and analysed by immunostaining with anti-chondroitin sulfate and anti-collagen type II antibodies according to method described above. The cultures were also analyzed by EM immunocytochemistry using anti-collagen type II.

\section{Osteogenesis}

Osteogenic differentiation was induced by culturing the hcADSCs (passage 12) for 2-3 weeks in differentiation medium composed of $10 \%$ FBS in $\alpha$ MEM supplemented with 50 $\mu \mathrm{g} / \mathrm{mL}$ gentamycin (Invitrogen), $10 \mathrm{mM} \beta$ glycerophosphate (Sigma), 10-8 M dexamethasone (Sigma), $50 \mu \mathrm{g} / \mathrm{mL}$ ascorbate (ICN). The medium was replaced three times a week. Osteogenic differentiation was confirmed by three methods: (i) Alkaline phosphatase activity staining, (ii) von Kossa staining, and (iii) Alizarin red S staining. For histochemical estimation of Alkaline phosphatase, cells were fixed with $4 \%$ formalin for $1 \mathrm{~h}$ at room temperature, washed three times with calcium- and magnesium-free PBS, and stained with BCIP-NBT stable mixture (5-Bromo-4chloro-3-indolylphosphate p-toluidine salt and nitroblue tetrazolium chloride mix substrate, Sigma) for $30 \mathrm{~min}$ at room temperature in the dark. Then the cells were rinsed and dried. This substrate solution yields a dark-colored precipitate in the presence of the phosphatase. Extracellular accumulation of calcium was assayed by von Kossa staining. The cells fixed in methanol for $2 \mathrm{~min}$ at $-20^{\circ} \mathrm{C}$ were incubated with freshly prepared $2 \%$ silver nitrate for $60 \mathrm{~min}$ under $60 \mathrm{~W}$ lamp, briefly rinsed with distilled water, and treated for 5 min with $2.5 \%$ sodium thiosulfate to remove un-reacted silver nitrate. Then the cells were washed with distilled water and air-dried. This staining allows revealing insoluble calcium salts in intercellular spaces. Mineralization of the extracellular matrix was also visualized by staining of the cultures with Alizarin red $\mathrm{S}$ adjusted by ammonium hydroxide to $\mathrm{pH} 4$. The cells were rinsed in PBS for 10 min, fixed by icecold $70 \%$ ethanol for $1 \mathrm{~h}$, and incubated in the $40 \mathrm{mM}$ Alizarin Red S for $10 \mathrm{~min}$ at room temperature followed by a wash with water.

Quantification and statistical analysis

After immunostaining with corresponding antibody, the percentage of differentiated cells
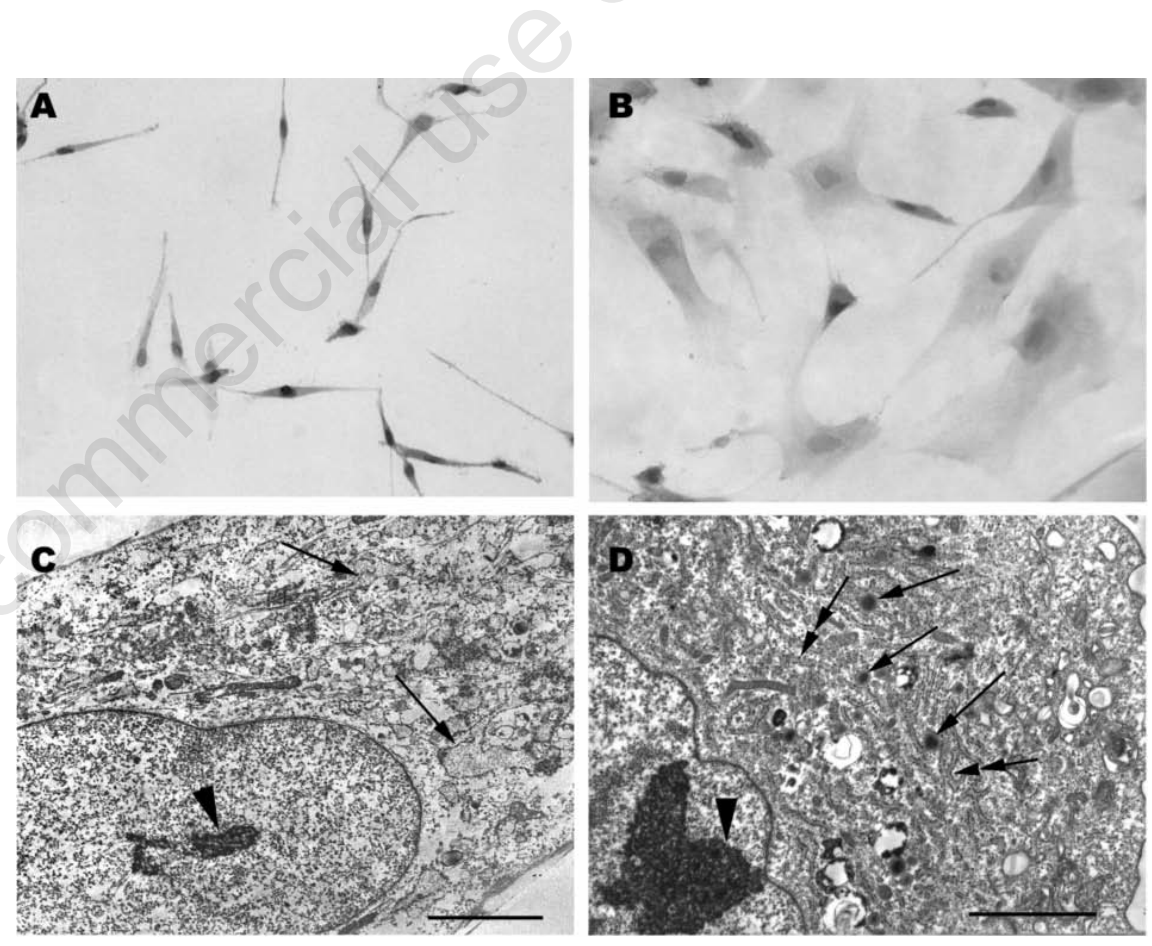

Figure 1. Behavior of human cardiac adipose tissue-derived stem cells (hcADSC) in culture. A, B) Bright-field micrographs illustrating morphology of the hcADSC cultures at passage 3 and 19, correspondingly. At early passage, cells maintain undifferentiated appearance and exhibit elongated, typical spindle-like shapes. At late passage, the cells increased in size and got flattened polygonal morphology. The cells were fixed with $4 \%$ PFA in PBS, and hematoxylin-eosin double stained; original magnification $\times 20$. C, D) Transmission electron micrographs showing ultrastructure of hcADSCs at passages 3 and 19, correspondingly. At early passage, the hcADSCs exhibited morphological features characteristic of non-differentiated cells; their nucleus contained a small nucleolus (arrowhead) and scanty cytoplasm in which the cytoplasmic organelles were poorly developed. Arrows point to dilated cisternae of RER. Late passage hcADSCs showed large nucleus with prominent nucleolus (arrowhead) and cytoplasm filled with many cisternae of rough endoplasmic reticulum (double arrows). The presence of secretory-like dark granules (arrows) was noted. Scale bars equal $1 \mu \mathrm{m}$. Abbreviation: hcADSCs, human subepicardial adipose tissue-derived stem cells; PBS, phosphate buffered saline; PFA, paraformaldehyde, RER, rough endoplasmic reticulum. 
(passages 19-25) was associated with an increase in the cell size and the appearing of large flattened cells which predominated (Figure 1B). The morphological characteristics of cultured hcADSCs at early and late passages were also examined by electron microscopy. The $3^{\text {rd }}$ passage hcADSCs, 0,4-0,6 $\mu \mathrm{m}$ at their short axes and $3-10 \mu \mathrm{m}$ at their long axes, possessed an undifferentiated phenotype (Figure 1C). They contained large, round or oval nucleus with a small nucleolus and finely dispersed chromatin particles, giving the nucleus a clear appearance. In the cytoplasm some number of mitochondria, free ribosomes, light vesicles, and small amounts of dilated cisternae of rough endoplasmic reticulum were observed. The passage 19 hcADSCs measured 1.7-14,5 $\mu \mathrm{m}$ in diameter. Their nucleus was slightly lobulated and contained a prominent nucleolus. The most evident feature of their cytoplasm was the presence of a large amount of rough endoplasmic reticulum (Figure 1D). The cytoplasm also characteristically contained scattered mitochondria and many small dense osmiophilic granules. Observed changes in the morphology of the hcADSCs during passaging testify to activation of protein synthesis.

\section{Karyotypic characteristics and pro- liferation}

Cytogenetic analysis revealed a normal diploid karyotype $46, \mathrm{XY}$ in $97 \%$ and $91 \%$ of the cells at culture passages 5 and 12 , respectively (Figure 2A).

At 400 band level of resolution, G-banding pattern of chromosomes in hcADSCs -corresponded to that in normal human cells. The loss of one chromosome in 3 out of 100 metaphase spreads at passage 5 and in two out of 100 metaphase spreads at passage 12 appears to be random. Found at passage 12 , all the seven cells with 47 chromosomes had three copies of autosome 2 (Figure 2B). Single chromatid breaks in 8q22 23, 14q13 21 and 16q22 in passage 5 cells and in $7 \mathrm{q} 32,9 \mathrm{q} 12$ (two cases) and 18q21.3 in passage 12 cells were observed. The number of polyploid cells during extended period in culture was found to increase from $2.8 \%$ (passage 5 ) to $9.6 \%$ (passage 12). In every tenth polyploid metaphase at passage 12, endoreplication of chromosomes was seen. In addition, passage 12 cells were characterized by nucleus polymorphism in shape and dimension.

We examined the proliferation status of the hcADSCs at different passages by immunohistochemistry with antibodies raised against PCNA - the protein synthesized in G1- and Sphase cells (Figure 3A).

The percentage of the cells with PCNA-positive nucleus at passages 6,10 and 19 comprised $86 \%, 75 \%$ and $56 \%$ of the total population, respectively. Thus, the rate of hcADSC proliferation progressively declines with the number of passages.

\section{Immunophenotypic characteristics}

The cell surface phenotype of hcADSCs at passages 5 and 16 has been analyzed using flow cytometry and antibodies specific for a range of surface markers. The collective data from these analyses are shown in Table 1 and the Supplementary file.

The cells at both passages were strongly pos-
A

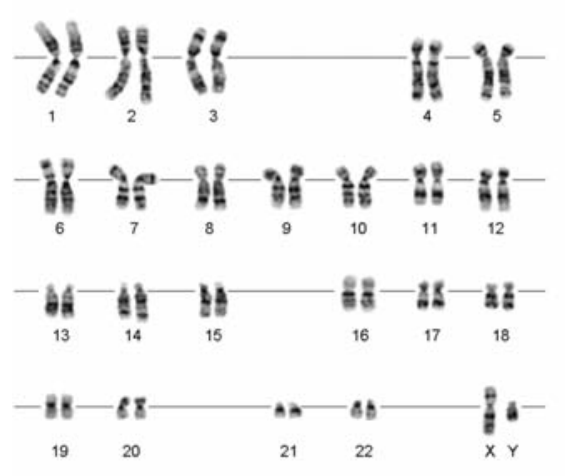

itive (more than 95\%) for CD 13, CD44, CD73 and CD105 antigens, and moderately positive for CD11b, CD45, CD71, CD90, CD130, and HLA class I antigens. They were negative (less than $0.5 \%$ ) for CD10, CD34, CD133, and HLA class II antigens. Statistically significant increase in the CD117 (about 95\% vs 71\%) positive populations and decrease in the CD41
Figure 2. Karyotype of human subepicardial adipose tissue-derived stem cells (hcADSCs) at early and late passages. When analysed by G-staining method, karyotype of hcADSCs shows a normal male karyotype $(46, \mathrm{XY})$ after 5 passages $(\mathrm{A})$, and abnormal karyotype with trisomy $2(47, X Y,+2)$ after 12 passages (B).
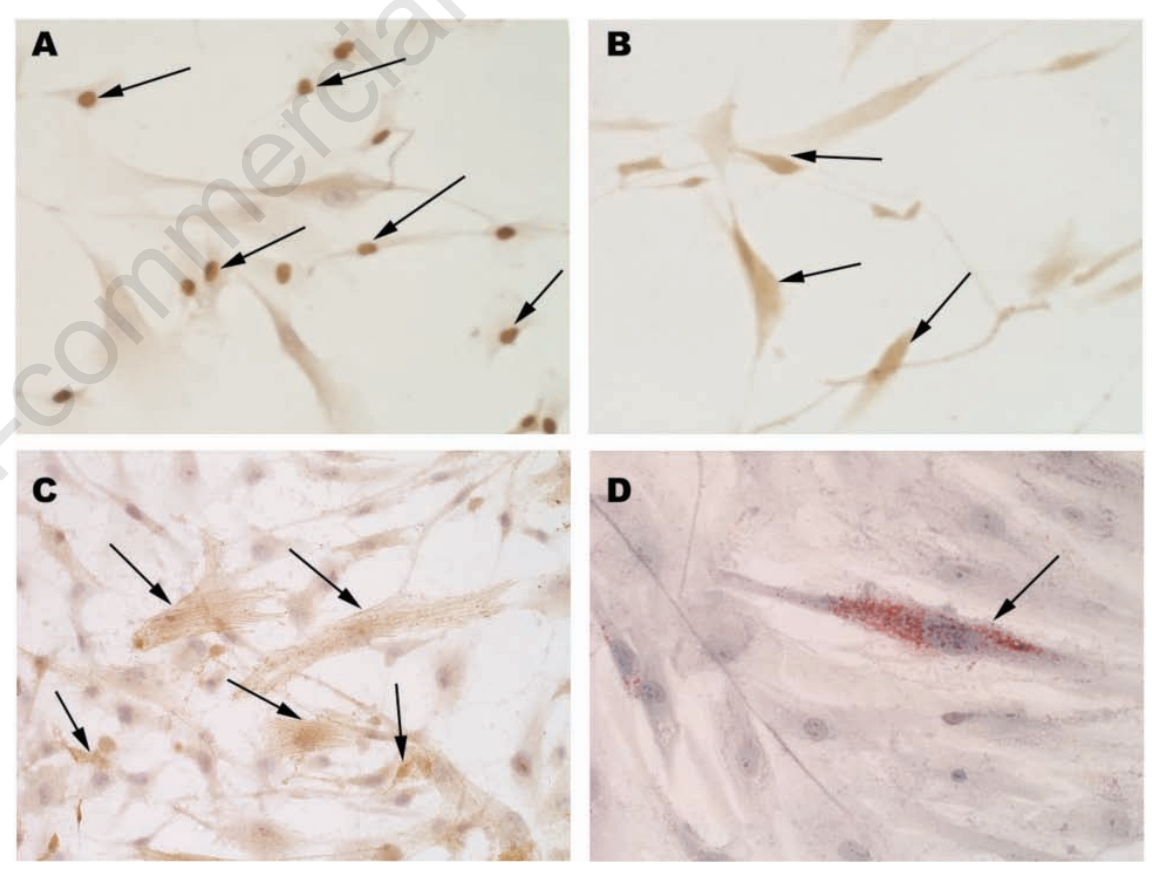

Figure 3. Spontaneous differentiation of hcATDCs. When passaged under basal conditions without any differentiation stimulation, hcATDCs actively proliferated and differentiated spontaneously towards endothelial, smooth muscle and adipose lineages. (A) This micrograph illustrates active passage 6 cell division reflected by PCNA-immunoreactive nuclei. Whereas at passage 6 hcATDCs differentiated mainly into VCAM-1-positive cells (B) and only few cells were positive for the antibody against hSMA, at passage 19 about the fifth part of the cells were hSMA-positive (C). Besides, Oil red O-positive cells (D) have appeared at late passage. Arrows point to PCNA (A), VCAM-1 (B), hSMA (C), and Oil red $O(D)$ - positive cells. Nuclei were counterstained with hematoxylin. Original magnification $\times 20$. Abbreviations: hcADSCs, human subepicardial adipose tissue-derived stem cells; hSMA, human smooth muscle actin; PCNA, proliferating cell nuclear antigen; VCAM-1, vascular cellular adhesion molecule-1; vWF, von Willebrand's factor. 
(about $0.5 \%$ vs $13 \%$ ) -positive population were observed at the later passage.

\section{Differentiation}

\section{Spontaneous differentiation at early and late passages}

To determine capacity of the hcADSCs for spontaneous differentiation, they were grown in culture medium without any differentiating agents. Three days after seeding at passages 6 and 19, the cells have been examined for expression of endothelial cell surface marker VCAM-1, smooth muscle surface marker hSMA, as well as for the 0il red 0 and von Kossa staining (Figures 3B-D). Passage 6 population contained $30.0 \pm 3.4 \%$ VCAM-positive cells and $5.0 \pm 2.8 \%$ hSMA-positive cells; both histochemical stainings gave negative results. At passage 19, VCAM was expressed by $31.7 \pm 5.2 \%$ of cells and hSMA by $21.0 \pm 0.8 \%$ of cells. Besides, $1.7 \pm 0.3 \%$ of this late passage hcADSCs were 0il red 0-positive. The population was negative for von Kossa staining. It can be seen that at the late passage the percent of the cells expressing endothelial cell marker shows no change, whereas the share of the cells expressing myogenic marker increased almost four times. In addition, adipogenic precursors have appeared.

We failed to observe spontaneous chondrogenic differentiation due to unsuitable culture conditions. That is, to induce chondrogenic response, the cells, apart from being transferred to differentiating medium, should be organized in 3D culture, namely, in pellets, while spontaneous differentiation has been

Table 1. Phenotypic characterization of human subepicardial adipose tissuederived cells at early and late passages.

\begin{tabular}{lcc} 
Surface marker & \multicolumn{2}{c}{ Positive cells (\%) } \\
Passage 5 & Passage 16 \\
CD10 & $<0.5$ & $<0.5$ \\
CD11b & $15.2 \pm 7.7$ & $59.7 \pm 17.7$ \\
\hline CD13 & $>95$ & $>95$ \\
CD34 & $<0.5$ & $<0.5$ \\
CD41 & $13.5 \pm 1.4$ & $<0.5$ \\
CD44 & $>95$ & $>95$ \\
\hline CD45 & $10.7 \pm 10.5$ & $6.4 \pm 3.2$ \\
CD71 & $<0.5$ & $1.32 \pm 0.14$ \\
\hline CD73 & $>95$ & $>95$ \\
CD90 & $10.0 \pm 1.7$ & $27.7 \pm 6.4$ \\
\hline CD105 & $>95$ & $>95$ \\
CD117 & $71.3 \pm 3.7$ & $>95$ \\
\hline CD130 & $9.9 \pm 7.6$ & $5.5 \pm 3.2$ \\
CD133 & $<0.5$ & $<0.5$ \\
\hline HLA-class I & $69.1 \pm 2.0$ & $42.7 \pm 6.0$ \\
HLA-class II & $<0.5$ & $<0.5$ \\
\hline
\end{tabular}

Data are presented as the mean \pm standard deviation $(n=3)$. examined in the typical monolayer cultures.

\section{Induced differentiations \\ Endothelial differentiation}

After 2-3 days in endothelial differentiation medium, the passage 6 hcADSCs were characterized as a network of endothelial-like cells (Figure 4A). They expressed a variety of endothelial lineage surface markers, such as CD31 (Figure 4C), vWF (Figure 4D), and VCAM-1 (Figure 4E).

Immunostaining showed $83.8 \pm 3.6 \%$ VCAMpositive cells, $73.8 \pm 0.6 \%$ CD31-positive cells, and $79.2 \pm 5.7 \%$ vWF-positive cells. In addition, $1.2 \pm 0.6 \%$ cells positive for hSMA (Figure 4B) were identified.

Thus, after endothelial differentiation induction, the percentage of differentiated endothelial cells was significantly higher, whereas the share of smooth muscle cells, on the contrary, was lower than these under spontaneous differentiation.

By seeding of hcADSCs on Matrigel-coated glass coverlips, as soon as after $18 \mathrm{~h}$ the formation of confluent monolayer of densely packed cells (Figure 4F) demonstrating VCAM-positivity was observed. Electron microscopy has
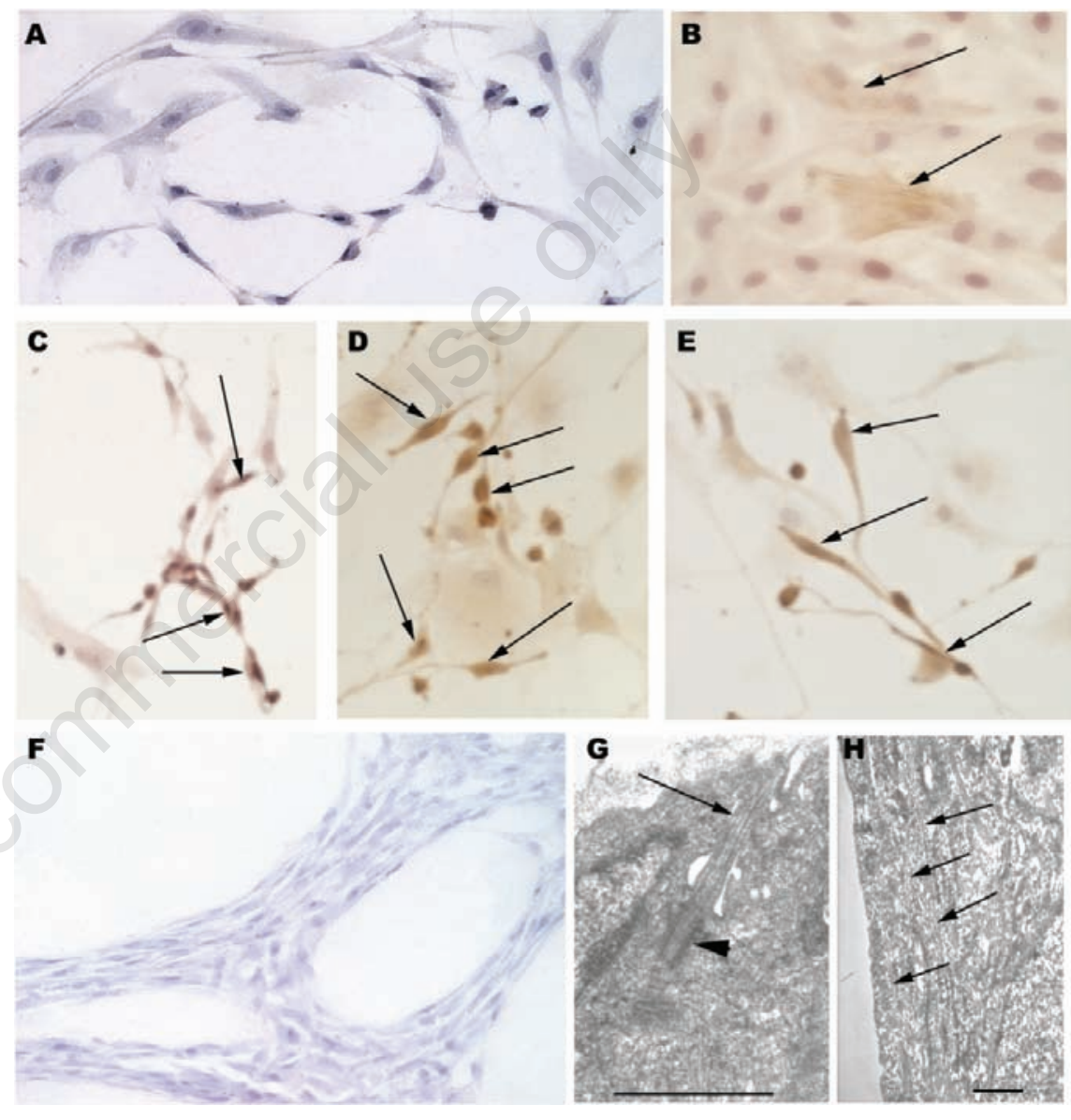

Figure 4. Endothelial and smooth muscle differentiation of hcATDCs. This figure shows results of culturing hcATDCs (at passage 6) under endothelial differentiation conditions. Two days after plating, most of the cells adopted a bipolar morphology, with long processes that formed a network between cells (A). Differentiated cells were positive for several endothelial cell markers: CD31 (C), vWF (D), and VCAM-1 (E). Some cells were immunoreactive for hSMA (B), but such cells were rare. Arrows indicate positively staining cells (stained in brown). The presence of Matrigel enhanced the differentiation. Cultures of hcATDCs grown on Matrigel with addition of endothelial differentiation factors formed single cell-thick contiguous monolayer sheet with large holes; cells are densely packed (F). Ultrastructural evidences of differentiation were observed in these cells. The culture contained occasional ciliated cells (G; arrow and arrowhead point to well formed cilium and basal body, respectively) and cells packed with numerous microfilaments (H; arrows). (A, F): The cells were fixed with $4 \%$ PFA in PBS, and hematoxylineosin double stained; original magnification $\times 20$. Bright-field micrographs: original magnification $\times 20$. Electron micrographs: scale bars equal $1 \mu \mathrm{m}$. Abbreviations: hcADSCs, human subepicardial adipose tissue-derived stem cells; hSMA, human smooth muscle actin; PBS, phosphate buffered saline; PFA, paraformaldehyde; VCAM-1, vascular cellular adhesion molecule-1; vWF, von Willebrand's factor. 
revealed some ultrastructural evidences of differentiation such as ciliary structures with a basal body (Figure 4G) and bundles of microfilaments (Figure $4 \mathrm{H}$ ), which are characteristic of mature endothelial cells.

\section{Adipogenic differentiation}

Culturing of hcADSCs in adipocyte differentiation medium resulted in their differentiation into 0il red 0-positive cells (Figure 5A). Ultrastructural analysis confirmed accumulation of a large number of intracellular lipid droplets which nearly or completely filled the cells (Figure 5B).

\section{Osteogenic differentiation}

Osteogenic induction during 2-3 weeks resulted in extracellular calcium accumulation confirmed by histology. The osteoblasts were positive for Alkaline phosphatase activity (Figure 5C), Alizarin red (Figure 5D), and von Kossa (Figure 5E) staining.

\section{Chondrogenic differentiation}

After 3 weeks of chondrogenic induction in vitro, hcADSC culture demonstrated depositions of extracellular matrix, which was histologically visualized by Alcian blue staining (Figure 6A). Immunostaining for type II collagen revealed its chondrogenic nature (Figure $6 \mathrm{~B})$. Ultrastructual observations also showed the formation of the typical structures characteristic of cartilage (Figure 6C). Immunogold labeling confirmed the presence of type II collagen within these cartilaginous constructs (Figure 6D).

\section{Discussion}

This study gives the histochemical, ultrastructural, proliferative, phenotypic, and karyotypic characteristics of the cells isolated from human subepicardial adipose tissue. It was shown that hcADSCs in vitro demonstrated properties of stem cells: they have the ability to both self-renew and multilineage differentiation. During long-term passaging, hcADSC population changed in morphology, phenotype, karyotype, and precursor cell composition. Low-passage cultures were found to spontaneously contain committed precursors of endothelial and smooth muscle cells. Late-passage cultures additionally contained precursors of adipocytes. With the number of passages the cells displayed a progressive decrease in proliferative capacity, which was consistent with their spontaneous differentiation. After an appropriate stimulation, hcADSCs additionally exhibited capacity to differentiate into osteocytes and chondrocytes.

Cytogenetic analysis revealed normal
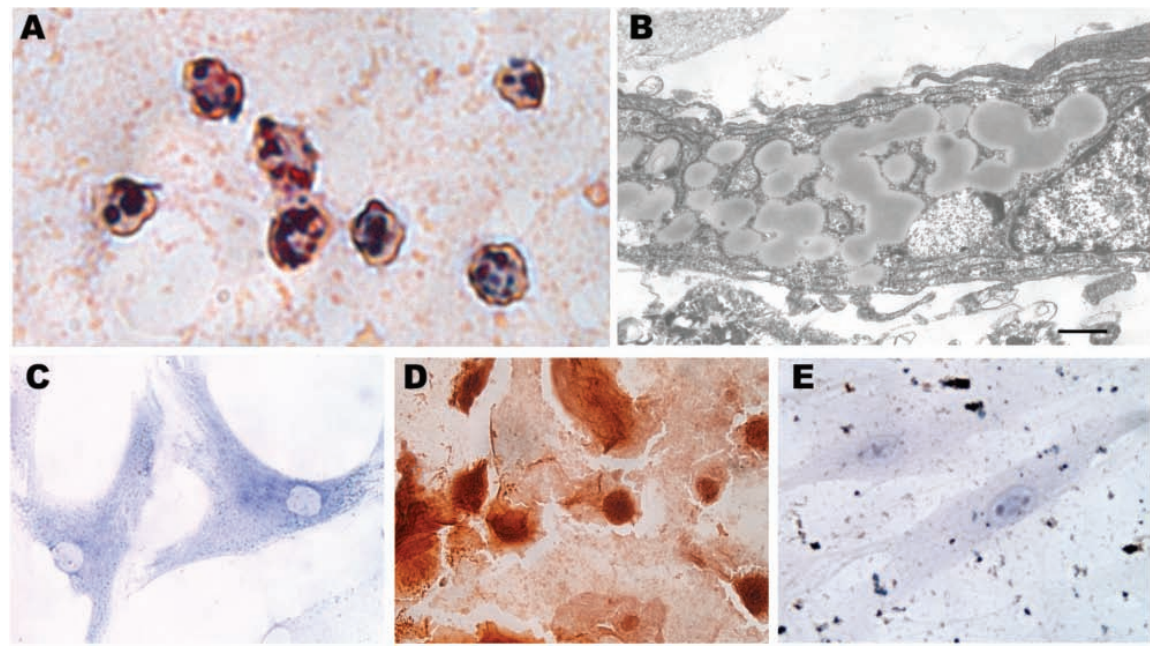

Figure 5. Adipogenic and osteogenic differentiation of human subepicardial adipose tissue-derived stem cells (hcATDCs). Three weeks after adipogenic stimulation, accumulation of lipid vacuoles in the cells was visualized under the microscope using Oil red $\mathrm{O}$ staining (A). Ultrastructural observation of these cells showed numerous intracellular oil droplets (B). When cultured during 2-3 weeks in osteogenic medium, hcATDCs produced depositions of calcium. The cells demonstrated high Alkaline phosphatase expression (C). The cultures were also positive for mineralization with Alizarin red (D; mineralized pericellular matrices are stained red) and von Kossa staining ( $\mathrm{E}$; calcium deposits interposed between cells are stained black) indicative for osteogenic differentiation. Bright-field micrographs: original magnification $\times 40$. Electron micrograph: scale bar equals $1 \mu \mathrm{m}$.
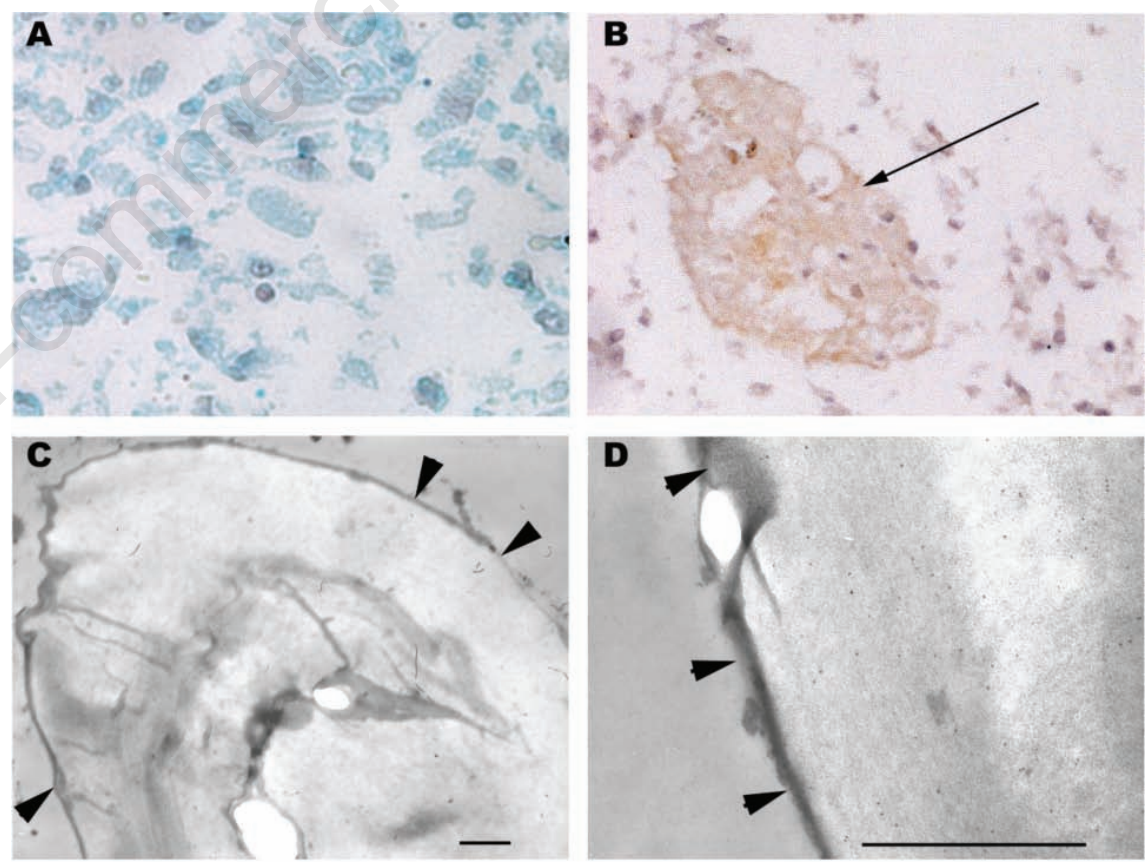

Figure 6. Chondrogenic differentiation of human subepicardial adipose tissue-derived stem cells. Three weeks after incubation in chondrogenic induction medium, human subepicardial adipose tissue-derived stem cells were fixed for light and electron microscopy. On paraffin sections, chondrogenesis was confirmed by Alcian blue staining (A), indicating glycosaminoglycan matrix production. Chondrogenic marker, collagen type II, in cartilaginous extracellular matrix (arrow) was detected by immunohistochemistry (stained in brown) (B). The cells were counterstained with hematoxylin to visualize nuclei. Original magnification $\times 20$. Ultrastructual observations also revealed typical cartilage structures (C) immunoreactive for collagen type II (D). The gold particles $(10 \mathrm{~nm})$ are distributed evenly above this structure. Arrowheads indicate the margin of the cartilage-like structures, Scale bars equal $1 \mu \mathrm{m}$. 
diploid karyotype in hcADSCs at low passage 5. However at high passage 12 , we observed appearance of a portion of aneuploid cells with recurrent trisomy 2 and increase of the polyploid cell number, which both are the signs of genome instability. Probably by itself trisomy 2 may provide proliferative advantages, so cells with this chromosome abnormality will expand. Trisomy 2 as the sole chromosomal abnormality has been shown in a number of human neoplasias: hepatoblastoma,20 myelodysplastic syndrome and acute myeloblastic leukemia, ${ }^{21}$ lymphoproliferative disorder post-liver transplant, ${ }^{22}$ and also in cultured cells of proliferative myositis. ${ }^{23}$ Since the aneuploidy is a hallmark of cancer, trisomy 2 may be the first step towards cell transformation in culture conditions and promote further karyotypic evolution of cells in vitro. Thus hcADSCs at early passages retained a genetic stability while high-passage cells exhibited a higher risk of genetic abnormalities such as chromosomal aneuploidy and multiploid karyotypes. Our data suggest that (i) application of cultivated hcADSCs for regenerative medicine is limited by the earliest passages and (ii) karyotypic examination of cultivated cells is required for their therapeutic application.

Comparative analysis of our flow cytometric results and available literature data has revealed a substantial overlap in the patterns of surface marker expression between hcADSCs and the MSCs from different loci. Like MSCs, ${ }^{24}$ hcADSCs have strongly expressed CD73 and CD105 and lacked the expression of CD34 and HLA class II antigens which indicates that hcATDC population was depleted of hematopoietic cells. Though CD34 is commonly identified as a marker for stem and progenitor cells of the hematopoietic system it is not restricted to them. Thus stem cells derived from adipose tissue of different localization differ in CD34 expression. Some studies in agreement with our data have reported them as CD34- negative cells, ${ }^{19,25,26}$ whereas other studies have reported them as CD34-positive cells..$^{10,12,27}$ It is possible that phenotypically different subpopulation of adipose tissue-derived stem cells do exist. However, the basis for these differences may also be related to methods used for cell isolation. It is still unclear whether these phenotypical differences reflect a distinct differentiation potential of these subpopulations.

The phenotype of hcADSCs was also similar to those of cells derived from adipose tissue of other depots in high expression of CD13, CD44 and CD105. ${ }^{10-12,19,25}$ However, in contrast to the surface marker profile of MSCs, ${ }^{24}$ and ADSCs from periumbilical and eyelid adipose tissues $^{12,13}$ and liposuction samples, ${ }^{26}$ hcADSCs were weakly positive for CD90 (proposed as a human MSCs marker). The same discrepancy was revealed with CD10 which expression was significantly lower on hcADSCs than on ADSCs from other localizations..$^{10,12}$ On the contrary, the expression of CD117 on hcADSCs was higher than that on periumbilical and eyelid ADSCs. ${ }^{12}$ Thus, hcADSCs show distinctive phenotypic features which suggests that they occupy their own stem cell niche.

So far, only one study has described the culture of mesenchymal stem cells isolated from human cardiac adipose tissue. ${ }^{16}$ The comparison of their and our data has revealed several distinctions in CD marker profile and differentiation capacities of cardiac adipose tissuederived cells investigated in these two studies. In contrast to the findings of these authors, we have revealed VCAM (CD106)-positive cells, and did not find a strong expression of CD90. Moreover, the authors have reported that hcADSCs did not display in vitro adipogenic potential. Under our conditions, we have found that hcADSCs have a capacity for adipogenic differentiation. Thus, although these two cultures of hcADSCs show many similarities, they also exhibit a number of distinctions. There might be several possibilities that account for this discrepancy. One is that the distinctions may merely be due to the variations in isolation and experimental procedures. Even small changes in culturing conditions could significantly influence the phenotypic and differential characteristics of cells. The other course of the revealed difference could derive from different locations in the organ with unique niche environment where from the tissue samples were taken. Conceivably distinct subpopulations of stem cells reside in cardiac adipose tissue, and the cells of each subpopulation have some unique characteristics in terms of phenotype and differentiation. One could propose that in vivo these subpopulations have somewhat different functions.

Recently, it has been shown that a stem cell pool from the same tissue source is composed of functionally diverse clones. Stem cells isolated from the samples of human subcutaneous adipose tissue has been separated into subpopulations that exhibited substantial distinctions in both the levels of surface marker expression and differentiation potential. ${ }^{28}$ It is evident that similarities and differences in subpopulations of adult hcADSCs should be taken into account at their use both in lab and in the clinic.

The study evaluated that hcADSCs can easily, without stimulation, spontaneously differentiate into endothelial cells, myocytes, and, at late passages, into adipocytes. Regarding the spontaneous differentiation, similar findings are reported for MSCs from rat bone marrow. ${ }^{29}$ Unstimulated culture of these cells was shown to contain the cells expressing myogenic, neurogenic and osteogenic markers. The ability to spontaneous differentiation in long-term culture was also detected for human embryonic30 and adipose tissue-derived ${ }^{31}$ stem cells.

Upon stimulation, the number of hcADSCs expressing appropriate specific markers significantly increased compared with that under spontaneous differentiation.

Thus, the stem cells resident in human cardiac adipose tissue demonstrated in vitro pronounced differentiation potential and spontaneously acquired endothelial, myogenic or adipogenic phenotype. Under the influence of appropriate differentiation stimulating factors these cells could also differentiate into osteocytes and chondrocytes. Obviously, an hcADSC culture presents a heterogeneous mixture of cells at least partially committed into multiple mesodermal lineages, including the myogenic, adipogenic, osteogenic, and chondrogenic, as well as into non-mesodermal lineage, such as endothelial cells.

It is tempting to speculate that the specific properties of resident subepicardial stem cells may be related to the rarity of the malignant cardiac tumors. Primary tumors of the heart (mainly sarcomas) are very uncommon..$^{32}$ The capacity of hcADSCs for facile spontaneous differentiation may be essential for a decreased risk of developing cancers in the heart. One of the cancer theories, based on a number of arguments, is that only uncommitted precursors are cellular targets for oncogenic events whereas committed progenitors are more limited in the neoplastic transformation..$^{33}$ Accordingly, tumorigenic transformation occurs at the stage of stem cell proliferation. It might have been proposed that after escaping from their specific niche, where external signals control quiescence, the partially committed hcADSCs very rapidly undergo differentiation into mature functional cells. Thereby uncontrolled proliferation of the cells and the time it takes for the influence of transforming agents are limited. To verify this proposal, further experiments including the comparative examination of the stem cells from subepicardial fat and from other adipose tissue depots under the same culture conditions need to be carried out. Possibly, due to their diminished tumorigenic potential, hcADSCs would be the better material for stem cell-based heart therapy than stem cell from other sources.

The hcADSCs being in close anatomical relation to coronary vessels most probably contribute to physiological renewal and revascularization following heart injury or an ischemic insult. In vitro these cells give rise to all the cue elements of the vascular wall, namely precursors for vascular endothelial cells, smooth muscle cells, perivascular adipocytes, and collagen producing chondrocytes. Our findings are in agreement with those obtained by the investigation of the subcutaneous abdominal fat-derived stem cells. ${ }^{34}$ The authors have shown that population of these cells present a mixture of vascular cellular precursors at vari- 
ous level of differentiation and that these cells can be induced additionally to differentiate into osteoblasts and chondrocytes.

Our results show that the subepicardial adipose tissue harbors the resident stem cell population presenting a mixture of undifferentiated and committed progenitor cells. Comparison of our data with those obtained on stem cells derived from adipose tissue of other localization revealed a number of distinct features of hcADSCs. Further research is required to learn more about the specific properties of these cells.

\section{References}

1. Prockop DJ. Marrow stromal cells as stem cells for nonhematopoietic issues. Science 1997;276:71-4.

2. Pittenger MF, Mackay AM, Beck SC, et al. Multilineage potential of adult human mesenchymal stem cells. Science 1999; 284:143-7.

3. Shih DT, Lee DC, Chen SC, et al. Isolation and characterization of neurogenic mesenchymal stem cells in human scalp tissue. Stem Cells 2005;23:1012-20.

4. Herrera MB, Bruno S, Buttiglieri S, et al. Isolation and characterization of a stem cell population from adult human liver. Stem Cells 2006;24:2840-50.

5. Young HE, Steele TA, Bray RA, et al. Human reserve pluripotent mesenchymal stem cells are present in the connective tissues of skeletal muscle and dermis derived from fetal, adult, and geriatric donors. Anat Rec 2001;264:51-62.

6. Lin HT, Chiou SH, Kao CL, et al. Characterization of pancreatic stem cells derived from adult human pancreas ducts by fluorescence activated cell sorting. World J Gastroenterol 2006;12:4529-35.

7. Beltrami AP, Cesselli D, Bergamin N, et al. Multipotent cells can be generated in vitro from several adult human organs (heart, liver, and bone marrow). Blood 2007;110: 3438-46.

8. Hoogduijn MJ, Crop MJ, Peeters AM, et al. Human heart, spleen, and perirenal fatderived mesenchymal stem cells have immunomodulatory capacities. Stem Cell Dev 2007;16:597-604.

9. Halvorsen YC, Wilkison WO, Gimble JM. Adipose-derived stromal cells -their utility and potential in bone formation. Int $\mathrm{J}$ Obes
Metab Disord 2000;24 Suppl 4:S41-4.

10. Gronthos S, Franklin DM, Leddy HA, et al. Surface protein characterization of human adipose tissue-derived stromal cells. J Cell Physiol 2001;189:54-63.

11. Zuk PA, Zhu M, Ashjian P, et al. Human adipose tissue is a source of multipotent stem cells. Mol Biol Cell 2002;13:4279-95.

12. Teplyashin AS, Tchupikova NI, Korzhikova SV, et al. Comparative analysis of cell populations with a phenotype similar to that of mesenchymal stem cells derived from subcutaneous fat. Tsitologiya 2005;47:63743. [Article in Russian, summary i n English].

13. Fischer LJ, Mcllhenny S, Tulenko T, et al. Endothelial differentiation of adiposederived stem cells: effects of endothelial cell growth supplement and shear force. $\mathrm{J}$ Surg Res 2009;152:157-66.

14. Toyoda M, Matsubara Y, Lin K, et al. Characterization and comparison of adipose tissue-derived cells from human subcutaneous and omental adipose tissues. Cell Biochem Funct 2009;27:440-7.

15. Rabkin SW. Epicardial fat: properties, function and relationship to obesity. Obes Rev 2006;8:253-61.

16. Bayes-Genis A, Soler-Botija C, Farré J, et al. Human progenitor cells derived from cardiac adipose tissue ameliorate myocardial infarction in rodents. $\mathrm{J}$ Mol Cell Cardiol 2010;49:771-80.

17. Ozkinay C, Mitelman F. A simple trypsinGimsa technique producing simultaneous G- and C-banding in human chromosomes. Hereditas 1979;90:1-4.

18. ISCN: An International System for Human Cytogenetic Nomenclature. LG Shaffer, MI Slovak, LJ Campbell (eds). Basel: S. Karger; 2009.

19. Cao Y, Zhao S, Liao L, et al. Human adipose tissue-derived stem cells differentiate into endothelial cells in vitro and improve postnatal neovascularization in vitro. Biochem Biophys Res Comm 2005;332:370-9.

20. Bardi G, Johansson B, Pandis N, et al. Trisomy 2 as the sole chromosomal abnormality in a hepatoblastoma. Gene Cromosomes Cancer 1992;4:78-80.

21. Czepulkowski B, Saunders K, Pocock C, et al. Mosaic trisomy 2 in myelodysplastic syndromes and acute myeloblastic leukemias. Cancer Genet Cytogenet 2004;145:78-81.

22. Ferro Delgado MT, Talavera M, GarciaMiguel P, et al. Trisomy 2 as the sole kary- otypic abnormality in a lymphoproliferative disorder post-liver transplant. Cancer Genet Cytogenet 2005;163:184-5.

23. Ohjimi $\mathrm{Y}$, Iwasaki $\mathrm{H}$, Ishiguro $\mathrm{M}$, et al. Trisomy 2 found in proliferative myosis cultured cell. Cancer Genet Cytogenet 1994; 76:157.

24. Dominici M, Le Blanc K, Mueller I, et al. Minimal criteria for defining multipotent mesenchymal stromal cells. The Internationsal Society for Cellular Therapy position statement. Cytotherapy 2006;8: 315-7.

25. Zhu Y, Liu T, Song K, et al. Adipose-derived stem cell: a better stem cell than BMSC. Cell Biochem Funct 2008;26:664-75.

26. Guan L, Li S, Wang Y, et al. In vitro differentiation of human adipose-derived mesenchymal stem cells into endothelial-like cells. Chinese Sci Bull 2006;51:1863-8.

27. Varma MJ, Breuls RG, Schouten TE, et al. Phenotypical and functional characterization of freshly isolated adipose tissuederived stem cells. Stem Cells Dev 2007;16:91-104.

28. Rada T, Reis RL, Gomes ME, et al. Distinct stem cells subpopulations isolated from human adipose tissue exibit different chondrogenic and osteogenic differentiation potential. Stem Cell Rev 2011;7:64-76.

29. Karaoz E, Aksoy A, Ayhan S, et al. Characterization of mesenchymal stem cells from rat bone marrow: ultgrastructural properties, differentiation potential and immunophenotypic markers. Histochem Cell Biol 2009;132:533-46.

30. Wang J, Lin G, Zhao HP, et al. Spontaneous differentiation of human embryonic stem cells into hematopoietic cells. Nan Fang Yi Ke Da Xue Xue Bao 2009;29:602-5.

31. Rubio D, Garcia-Castro J, Martin MC, et al. Spontaneous human adult stem cell transformation. Cancer Res 2005;65:3035-9.

32. Rica DC, Reardon MJ. Left heart sarcomas. Methodist Debakey Cardiovasc J 2010;6: 49-56.

33. Wang JCY. Good cells gone bad: the cellular origins of cancer. Trends Mol Med 2010;16:145-51.

34. Lin G, Garcia M, Ning H, et al. Defining stem and progenitor cells within adipose tissue. Stem Cells 2008;17:1053-63. 